\title{
La rénovation de la gestion des carrières dans le secteur public à l'épreuve des relations entre État et groupes d'intérêt. Le cas des Conseillers Techniques Sportifs du Ministère des Sports
}

Human Resources Management Renovation in the Public Sector in the Test of the Relations between State and Interest Groups. The Case of Agents of the Sports Ministry

Marina Honta et Samuel Julhe

\section{(2) OpenEdition} Journals

Édition électronique

URL : http://journals.openedition.org/pmp/7065

ISSN : 2119-4831

\section{Éditeur}

Institut de Management Public (IDPM)

\section{Édition imprimée}

Date de publication : 15 avril 2014

Pagination : 239-257

ISBN : 978-2-7430-1997-6

ISSN : 0758-1726

\section{Référence électronique}

Marina Honta et Samuel Julhe, «La rénovation de la gestion des carrières dans le secteur public à l'épreuve des relations entre État et groupes d'intérêt. Le cas des Conseillers Techniques Sportifs du Ministère des Sports », Politiques et management public [En ligne], Vol 31/2 | 2014, mis en ligne le 31 décembre 2016, consulté le 20 avril 2019. URL : http://journals.openedition.org/pmp/7065 


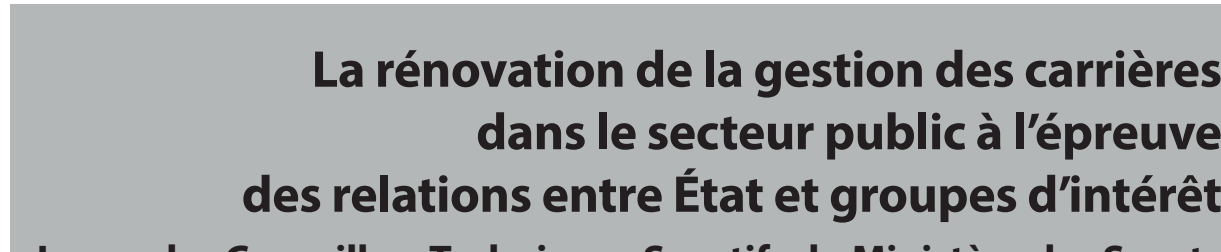

Le cas des Conseillers Techniques Sportifs du Ministère des Sports

Marina Honta ${ }^{a, b^{*}}$ et Samuel Julhe ${ }^{b}$

${ }^{a}$ Chercheuse associée au Centre Émile Durkheim (UMR 5116-CNRS), 33000 Bordeaux

'Université de Bordeaux, LACES, EA 4140, 33000 Bordeaux

\section{Résumé}

Les réformes de modernisation de l'État font de la rénovation de la gestion des ressources humaines l'une des composantes majeures de la "performance » désormais attendue de la puissance publique. À partir du cas des Conseillers Techniques Sportifs (CTS), agents du Ministère des Sports, nous montrons que les dispositions qu'elles prévoient restent limitées tant les principes structurant les rapports entre l'État et les fédérations sportives heurtent les axes des réformes adoptées. La position administrative des CTS et leur contexte relationnel de travail exacerbent en effet les tensions et paradoxes inhérents à la mise en œuvre d'une démarche d'appréciation des professionnels. La diversité des acteurs en présence, aux intérêts pouvant être divergents, oriente actuellement la définition de leur parcours professionnel et suscite par là même des sentiments d'injustice et d'iniquité.

๑ 2014 IDMP/Lavoisier SAS. Tous droits réservés

Mots clés : gestion des ressources humaines, Conseillers Techniques Sportifs, réforme de l'État, carrière.

\section{Abstract}

Human Resources Management Renovation in the Public Sector in the Test of the Relations Between State and Interest Groups. The Case of Agents of the Sports Ministry. Recent reforms to modernize the State are the renovation of the management of human resources one of the major components of the "performance" now expected of public power. From the case of "Conseillers Techniques Sportifs" (CTS), agents of the Ministry of Sports, we show that the provisions are limited as they provide the principles underpinning the relationship between the State and the sports federations face the axes of the reforms. Administrative position of CTS and relational context of work indeed exacerbate tensions and paradoxes inherent in the implementation of a process of assessment professionals. The diversity of actors, interests may be divergent,

\footnotetext{
*Auteur correspondant : marina.honta@u-bordeaux.fr
}

doi :10.3166/pmp.31.239-257 @ 2014 IDMP/Lavoisier SAS. Tous droits réservés 
currently directs the definition of their careers and thereby creates feelings of injustice and iniquity.

(c) 2014 IDMP/Lavoisier SAS. Tous droits réservés

Keywords: Human resources management, Agents of the Sports Ministry, State reform, career.

\section{Introduction}

Depuis une trentaine d'années en France le rôle de la puissance publique et le périmètre de son action font l'objet d'une réévaluation critique : la taille de l'administration, son organisation, son coût rapporté aux services effectivement rendus et les règles applicables à la fonction publique sont au centre de débats alimentant les projets de réforme (Dreyfus, 2006).

Confronté à cette nouvelle configuration, le système administratif français a, comme dans d'autres pays (Talbot, 2003 ; Giauque et Caron, 2004 ; Bourgon, 2009; Massey, 2010), fait l'objet de multiples programmes visant la « modernisation de l'État ». Initialement expérimental et peu publicisé dans les années 1990 (Bezès, 2008), ce processus est systématisé au sein de la loi organique relative aux lois de finances (LOLF) ${ }^{1}$ puis dans le cadre de la révision générale des politiques publiques (RGPP) et désormais de la modernisation de l'action publique (MAP). Toutes ces réformes font de l'accroissement de la crédibilité de l'État par un meilleur pilotage des politiques publiques et de la rénovation de la gestion des ressources humaines des composantes majeures de ce processus.

Ce contexte de renforcement des formes de légitimation des politiques publiques à l'aune de leur efficacité et efficience ne pouvait en effet manquer d'influer sur le déroulement des carrières des professionnels d'autant plus qu'en France, la question de la gestion des ressources humaines au sein de la fonction publique d'État fait l'objet de plusieurs critiques depuis une quinzaine d'années. En ce sens, de nombreux rapports ont souligné sa situation dégradée et l'ont jugée, par ailleurs, rigide et inadaptée aux enjeux actuels (Vallemont, 1999; Pochard, 2003 ; Silicani, 2008; Chemla-Lafay, 2008; Pêcheur, 2013). Les transformations, exprimées dans ces travaux et progressivement engagées, découlent également de l'influence de l'Union Européenne promouvant un modèle de fonction publique d'emploi à orientation professionnelle et emplois ouverts distinct de la structuration historique française en fonction publique de carrière et emplois fermés structurés par corps (Bezès, 2008; Lemoyne de Forges, 2009).

Ainsi, sans que les dispositions législatives et réglementaires relatives au statut des fonctionnaires soient réformées, la LOLF puis la RGPP ont souhaité concilier ce dernier avec une approche plus individualisée des carrières. L'enjeu est de permettre de renseigner les motivations de l'agent, de recenser ses compétences et ses besoins en formation pour accéder aux postes ouverts dans le cadre de la mobilité en fonction de ses projets personnels mais aussi de l'appréciation qui est portée sur lui. La gestion collective des carrières fait place à une différenciation des trajectoires professionnelles s'appuyant sur les compétences et l'expérience du fonctionnaire et l'incitant, ce faisant, à rechercher l'efficacité (Lapin, 2009). Derrière cette idée se trouve le projet de caractériser, non plus le poste occupé, mais les capacités des individus et leur manière de se comporter en situation (Jeannot, 2005). Dans ces conditions, l'appréciation

'Loi n²001-692 du $1^{\text {er }}$ août 2001. 
des agents est la conséquence individuelle de l'assignation d'objectifs aux administrations par les divers programmes de modernisation. En ce sens, une réforme de leur évaluation a été adoptée, celle-ci reposant sur le principe de l'avancement au mérite sur la base d'entretiens professionnels afin de mieux apprécier « leur valeur individuelle $»^{2}$.

Dans ce contexte, s'intéresser aux Conseillers Techniques Sportifs (CTS), cadres du Ministère des Sports qui ont pour prérogatives de promouvoir le sport à tous les niveaux, d'organiser la sélection et d'entraîner les équipes nationales, de découvrir les espoirs et de former les entraîneurs, revêt plusieurs intérêts.

Malgré l'instauration progressive d'une «boîte à outils » de rénovation de la gestion des ressources humaines inspirée des méthodes adoptées dans le secteur privé se diffusant dans l'ensemble des pays de l'OCDE, les pratiques à l'œuvre et les avancées en la matière demeurent très disparates d'un État et d'un Ministère à l'autre (Horton et al., 2002; Pochard, 2003 ; Pollitt et Bouckaert, 2004; Giauque et Caron, 2004). Parce que les usages et les effets de ce processus de modernisation sont hétérogènes voire paradoxaux, s'attacher à analyser spécifiquement comment ce dernier est vécu par ceux qu'il concerne permet de participer aux débats théoriques et empiriques contemporains sur ce sujet (Aucoin, 1990; Ferlie et al., 1996; Hood et Lodge, 2004; Jeannot, 2005; Pochard, 2008; Lapin, 2009; Chanut et Rojot, 2011).

Au même titre ensuite que d'autres corps d'agents publics tels que les conservateurs de musées (Octobre, 1999), la faiblesse de l'effectif des CTS, un peu plus d'un millier, et leur moindre visibilité sociale comparée à celle des enseignants, des policiers ou des professionnels de santé, en ont fait un objet d'étude moins immédiat. Pourtant, ces agents témoignent du volontarisme de l'État dans le secteur sportif. Si l'implication des pouvoirs publics en la matière est une réalité qui touche de nombreux pays, le modèle français se caractérise effectivement par un degré élevé et des formes variées d'intervention publique. Il consacre la position se voulant longtemps dominante du Ministère des Sports qui associe les fédérations sportives, délégataires de puissance publique et placées sous sa tutelle, à la fabrication et à la mise en œuvre de cette politique publique. Ce faisant, l'État permet aussi à ces acteurs de disposer de ressources publiques et notamment de personnels que sont les CTS. En étant « placés auprès » des fédérations sportives (et de leurs instances territoriales, les ligues ou comités régionaux), ces agents occupent une place prépondérante dans les axes du partenariat entre le Ministère et le mouvement sportif, axes désormais sous tensions.

Ainsi, après avoir précisé les changements prévus par les diverses réformes de modernisation de l'État sur la gestion des parcours professionnels des agents publics, nous montrons que leurs effets restent limités s'agissant des CTS tant les principes structurant les rapports entre le Ministère des Sports et les fédérations sportives résistent et heurtent les axes des réformes adoptées. La position administrative des CTS et leur contexte relationnel de travail exacerbent en effet les tensions et paradoxes inhérents à la mise en œuvre d'une démarche d'appréciation des professionnels (Trépo et al., 2002; Baudry et Dubrion, 2005; Garant et al., 2010). La diversité des acteurs en présence, aux intérêts pouvant être divergents, oriente actuellement la définition de leur parcours professionnel et suscite par là même des sentiments d'injustice et d'iniquité.

${ }^{2}$ Décret $n^{\circ} 2002-682$ du 29 avril 2002 relatif aux conditions générales d'évaluation, de notation et d'avancement des fonctionnaires de l'État. Arrêtés du 13 février 2006 relatifs aux modalités de notation de ces personnels. 


\section{Les principes de la gestion des carrières des CTS dans le cadre des réformes de modernisation de l'État}

Dans le cadre de droit commun devant organiser la gestion des parcours professionnels des agents de la fonction publique, plusieurs textes précisent les modalités spécifiques de celle des CTS. Cette dernière a progressivement été réglementée afin d'asseoir plus particulièrement leur position administrative. Afin que le Ministère chargé des Sports conserve la gestion des carrières de ces agents, les CTS sont « placés auprès » (et non « mis à disposition » ou « détachés ») des fédérations sportives ou de leurs instances territoriales et occupent à ce titre une position singulière voire unique au sein de la fonction publique d'État.

\subsection{Les modalités officielles de la gestion des carrières des CTS}

Le modèle français d'organisation du sport construit à partir de la fin de la Seconde Guerre Mondiale repose sur un partenariat, en bien des points singulier en Europe, entre l'État et le mouvement sportif. Plus généralement, cette singularité renvoie au fait que la France est souvent considérée comme difficile à situer dans la classification dichotomique des modes de relations entre État et société connus en Europe, les modèles pluraliste et néocorporatiste. Ces derniers varient fortement concernant le rôle de l'État, les capacités d'organisation et de mobilisation des intérêts économiques et sociaux et en ce qui concerne les formes de coopération entre l'État et ces intérêts. Chacun renseigne à la fois sur les stratégies dominantes des groupes d'intérêt et des mouvements sociaux et sur l'attitude de l'État face à ces acteurs (Grossman, 2007, p. 157). Au regard de cette difficulté à positionner la France dans cet idéal-type, le modèle du « corporatisme sectoriel », ou de « l'étatisme » est mobilisé pour caractériser «l'État en action » dans ce pays (Jobert et Muller, 1987). Convoqué pour analyser ces interactions dans le secteur de l'agriculture notamment, il renvoie également aux modes de relations rencontrés dans le champ sportif, modes de relations désormais contestées par le mouvement sportif. En ce sens, par la procédure de délivrance de l'agrément et de la délégation aux fédérations sportives faisant suite à leur adoption de statuts conformes à des orientations obligatoires, l'État leur confère la responsabilité de gérer, en toute indépendance, une ou plusieurs disciplines sportives. Ces principes, fixés sur les plans législatifs et réglementaires et qui pourraient prochainement être révisés ${ }^{3}$, prévoient également que les fédérations restent placées sous la tutelle du Ministère des Sports qui conserve un droit de regard sur leurs activités. Au moyen, entre autres, de ces interactions, l'État a par ailleurs souhaité produire et diffuser, dès le début des années 1960, sa conception d'un service public du sport. Les axes du partenariat visent ainsi à maintenir voire à améliorer le rang de la France dans le classement des meilleures nations sportives. Ils ont également pour objet le développement d'une pratique sportive de qualité et diversifiée pour le plus grand nombre sans recherche systématique de performance. Responsables de la mise en œuvre de ces orientations, les fédérations sportives reçoivent un soutien de l'État. Les CTS, qui relèvent du corps des professeurs de sport depuis 1985, représentent alors une

${ }^{3}$ Largement controversée désormais par les dirigeants des fédérations sportives, cette tutelle devait être supprimée dans la future loi de modernisation de la gouvernance et de l'organisation du sport. Les remaniements ministériels suite aux élections municipales de 2014 et les concertations actuelles entre la nouvelle Ministre, Najat Vallaud-Belkacem, et le mouvement sportif laissent désormais planer le doute quant à la suite à donner à ce projet de loi qui pourrait être « enterré ». 
ressource afin qu'elles les exercent et leurs missions s'inscrivent désormais dans les quatre axes du « Programme Sport » (au sens de la LOLF) : la promotion du sport pour le plus grand nombre, le développement du sport de haut niveau, la prévention par le sport et la protection des sportifs, la promotion des métiers du sport.

Plus généralement, leur récit biographique révèle qu'exercer professionnellement dans le domaine du sport trouve ses racines dans un parcours entamé de façon précoce, durant l'enfance ou l'adolescence, avec une spécialisation très rapide dans une discipline sportive. L'entrée dans le métier est le fruit d'un long processus d'incorporation d'un goût pour les activités physiques, le plus souvent initié par le père et/ou un frère, et d'acculturation au monde sportif comme pratiquant(e), puis compétiteur ou compétitrice, suivi du réajustement d'une vocation pour les métiers de l'encadrement des activités physiques. Cette socialisation sportive précoce conduit les interviewés à privilégier également des études dans le domaine sportif. Sur ce point, les CTS sont généralement titulaires d'un diplôme délivré soit par le Ministère de l'Enseignement Supérieur et de la Recherche (filière en Sciences et Techniques des Activités Physiques et Sportives) soit par le Ministère chargé des Sports. La possibilité de passer le concours du professorat de sport nécessite, elle, que ces personnes soient titulaires d'un diplôme de niveau II délivré par ces mêmes instances ${ }^{4}$.

Une fois recrutés et comme tout agent de la fonction publique, les CTS sont rémunérés selon une grille indiciaire. En fonction de la classe et de l'échelon considérés, leur rémunération brute mensuelle s'élève entre 1600 euros et un maximum de 3600 euros.

Ces professionnels ensuite, rassemblés sous l'appellation générique de CTS, se répartissent en différentes catégories en fonction des missions qu'ils se voient confier ${ }^{5}$ :

- le Directeur Technique National (DTN) concourt à la définition de la politique sportive fédérale, veille à sa mise en œuvre et contribue à son évaluation;

- les Conseillers Techniques National ou Régional (CTN ou CTR) mènent, respectivement l'un au niveau national et l'autre au niveau territorial, des tâches d'observation et d'analyse, de conseil et d'expertise, d'encadrement de sportifs, de formation des cadres, d'organisation et de développement de l'activité sportive de la fédération intéressée;

- l'Entraîneur National (EN) encadre les membres des équipes de France et participe à l'animation de la filière du sport de haut niveau de la fédération.

Il n'existe officiellement aucune relation hiérarchique entre ces diverses catégories de CTS. Qu'ils exercent des missions exclusivement nationales ou régionales ou les deux, ces agents sont placés sous l'autorité hiérarchique du Directeur des Sports au Ministère ou du Directeur Régional de la Jeunesse, des Sports et de la Cohésion Sociale (DRJSCS) qui établit, après avis des intéressés, leur lettre de mission à partir des propositions produites par le DTN. Dans le cas où l'agent est CTR, l'avis du président de la ligue ou du comité régional est également requis. Le Directeur régional assure, en outre, leur gestion administrative en lien avec l'administration centrale, organise, anime leur regroupement trois fois par an et procède à leur évaluation individuelle.

${ }^{4}$ Une Licence en STAPS ou un Brevet d'État d'Éducateur Sportif (BEES) du second degré ou un Diplôme d'État Supérieur de la Jeunesse, de l'Education Populaire et du Sport (DESJEPS).

${ }^{5}$ Circulaire nDS/DSA1/DRH/DGPJS/2011/37 du 28 janvier 2011 relative aux modalités d'intervention des personnels exerçant les missions de conseillers techniques sportifs (CTS) auprès des fédérations sportives. Tous les DTN et EN notamment ne sont pas des agents de l'État. Ils peuvent également être recrutés par les fédérations sur ressources propres. 
Concernant cette dernière, si les réformes de modernisation engagées prévoient le principe de l'avancement au mérite ${ }^{6}$ sur la base de l'entretien individuel, dans le cas des CTS, c'est encore celui de leur notation par le Ministre chargé des Sports sur proposition exclusive du directeur de leur service d'affectation qui prévaut ${ }^{7}$. Toutefois, les diverses notes administratives annuelles précisant aux chefs de service les modalités de cette évaluation, insistent elles aussi sur la nécessaire gestion individuelle des carrières. Elles rappellent en effet que l'attention doit particulièrement porter sur les critères d'appréciation et les modalités d'attribution de la note lesquels permettent d'assurer aux intéressés, conformément à leur statut, un avancement différencié compte tenu de leur manière de servir ${ }^{8}$.

En outre, la durée des missions exercées par les CTS ne peut excéder quatre ans. Leur renouvellement à l'issue de la durée fixée est opéré après avis du DTN ou, en l'absence de ce dernier, du président de la fédération. Néanmoins, le cadre législatif et réglementaire rend également possible une interruption de mission avant le terme soit à l'initiative du Ministre des Sports ou à la demande de l'agent ou du président de la fédération. Les motifs doivent alors être fondés sur l'intérêt du service (la restructuration nécessaire de l'organigramme de la direction technique nationale pour faire face à la concurrence sportive internationale et aux exigences de la performance, la modification du contenu et/ou de la répartition des missions des CTS auprès de la fédération sportive intéressée, le non-respect avéré des objectifs figurant dans la lettre de mission, une situation de conflit durable). Dans ce cas de figure, il appartient au chef de service concerné de confier, à titre provisoire, de nouvelles missions à un CTS qui n'est plus en mesure d'exercer les siennes, dans l'attente du règlement de sa situation. L'agent dont la mission a été interrompue reçoit une nouvelle affectation dans le respect des règles fixées par son statut ${ }^{9}$.

Les CTS, enfin, sont eux aussi concernés par l'augmentation des procédures de suivi de leur activité prises dans le cadre des réformes de modernisation en étant confrontés à un resserrement du contrôle exercé par les services de l'État. Un instrument de « gouvernement à distance » récemment élaboré (le « CTS Web»), vise ainsi à « homogénéiser les lettres de mission, faciliter la transmission de ces documents et ainsi mieux gérer les CTS et leur parcours professionnel $»^{10}$.

\subsection{Méthodologie}

Si plusieurs travaux ont analysé les changements que les divers programmes de modernisation de la gestion publique ont entraînés sur les politiques d'intervention de l'État dans

\footnotetext{
${ }^{6}$ La classe normale des professeurs de sport comporte onze échelons, la hors classe 7 . Leur carrière est régie par des principes de droit commun à l'ensemble des agents de la fonction publique d'État. Outre l'avancée à l'ancienneté, ils peuvent en fonction de leur notation, être proposés au petit ou au grand choix par leur supérieur hiérarchique pour accélérer leur passage à l'échelon supérieur. Ils peuvent également candidater aux postes ouverts à la mutation.

${ }^{7}$ Note de service $\mathrm{N}^{\circ} \mathrm{DRH} / \mathrm{DRH} 1 \mathrm{C} / 2013 / 278$ du 10 juillet 2013 relative à la notation 2013 des personnels techniques et pédagogiques : professeurs de sport, conseillers et chargés d'éducation populaire et de jeunesse.

${ }^{8} \mid \mathrm{d}$ dem, p. 4. Cette manière de servir est appréciée au regard de sept critères : sens du service public, efficacité professionnelle, autorité professionnelle et rayonnement, investissement professionnel, aptitude au dialogue avec les partenaires, qualité d'analyse et d'expertise, sens de l'initiative.

${ }^{9}$ Circulaire nDS/DSA1/DRH/DGPJS/2011/37 op.cit.

${ }^{10}$ Idem.
} 
le domaine sportif en France comme dans d'autres pays (Honta, 2009; Houlihan et Green, 2009), ils n'étudient pas leur impact sur les modalités vécues de la gestion des parcours professionnels des agents publics. Or, parce que les réformes du secteur public sont porteuses de paradoxes, de démotivation, de méfiance mais aussi de gains pour ces derniers (Ferlie et al., 1996; Giauque et Caron, 2004 ; Chanut et Rojot, 2011), nous avons souhaité appréhender ces effets à partir des perceptions des CTS tout en les replaçant dans un contexte plus large d'évolution des rapports entre l'État et les fédérations sportives françaises.

À ce titre, 44 entretiens ont été effectués auprès de :

- 39 CTS dont 18 exerçant des missions régionales et représentant un large panel de disciplines sportives (athlétisme, aviron, badminton, canoë-kayak, football, gymnastique, Éducation Physique et Gymnastique volontaire, handball, handisport, judo, karaté, pelote basque, rugby, surf, tir) ${ }^{11}$,

- 5 représentants d'organisations syndicales défendant les intérêts de ce groupe professionnel.

Ces entretiens, d'une durée moyenne d'une heure et quarante cinq minutes, visaient à aborder les points suivants : 1) les trajectoires sociales des CTS ; 2) l'explicitation du faisceau de tâches qu'ils effectuent ; 3) les modalités effectives de gestion de leur parcours professionnel ; 4) le rapport que les CTS entretiennent vis-à-vis du rôle qui leur est assigné, de ses éventuelles transformations, plus généralement de leur statut d'agent de la fonction publique d'État; 5) les formes et enjeux de la mobilisation des organisations syndicales à l'égard des réformes relatives à la gestion de leur carrière engagées et plus globalement du processus de modernisation de l'État.

La retranscription intégrale de ces entretiens et leur analyse par la méthode thématique, ont permis que soit livré - au croisement d'éléments objectifs et subjectifs - le sens que les CTS accordent à leur travail, à la manière avec laquelle est gérée leur carrière et aux diverses mesures adoptées dans le cadre des réformes de modernisation de l'administration. Cette option consistant à apprécier la façon dont les agents eux-mêmes appréhendent leur situation professionnelle, dimension essentielle du rapport au travail, a été réalisée sans méconnaître la difficulté à cerner la nature de ce rapport à partir de récits subjectifs (Méda, 2010).

Cette analyse des entretiens a été complétée par une exploitation, à partir des mêmes thèmes, de diverses sources documentaires à des fins de recoupement : presse nationale, autres recherches effectuées sur ces objets, bulletins syndicaux, débats au Parlement, rapports publics nationaux, discours ministériels, analyse de contenu des textes législatifs et réglementaires relatifs au cadre d'emploi des CTS.

\section{Les avatars de la gestion personnalisée des parcours professionnels des CTS}

Les réformes de modernisation de l'État destinées à assurer une meilleure individualisation des parcours professionnels des agents publics n'ont guère d'impact sur celle des CTS. En tant qu'agents publics placés auprès de structures privées et ce faisant d'une grande diversité d'acteurs, les modalités concrètes de gestion de leur carrière (établissement de leur lettre de mission, modes d'évaluation, de mobilité et de rémunération) échappent pour partie à l'administration et sont exposées à la forte hétérogénéité des contextes relationnel

\footnotetext{
11En 2014, 1660 CTS sont placés auprès des diverses fédérations sportives.
} 
et organisationnel de travail. Ces caractéristiques, perçues comme des incohérences et dysfonctionnements, sont à l'origine de nombreuses insatisfactions .

\subsection{Une injonction à la mobilité et un faible contrôle des trajectoires professionnelles}

S'agissant tout d'abord de la définition des missions des CTS, les entretiens ont révélé une grande hétérogénéité des pratiques dans la formalisation de cette opération. Par ailleurs, ce processus témoigne clairement de la position dominante du DTN, le Directeur régional entérinant le plus souvent les propositions formulées.

Concernant l'exercice de ces missions, les CTS interrogés insistent tous sur l'importance du volume de travail qu'ils effectuent en raison d'horaires atypiques, de déplacements fréquents pour encadrer et suivre des athlètes notamment en stages et compétitions. Bien qu'ils disposent d'une grande autonomie pour organiser leur travail, symbole par excellence des emplois de cadres et de leurs prérogatives (Cousin, 2008), ces contraintes sont exacerbées lorsque les CTS cumulent missions régionales et nationales. Cette situation les expose par ailleurs aux attentes aussi bien du DTN que des dirigeants sportifs nationaux et régionaux ce qui non seulement amplifie les difficultés pour toutes les assumer mais les amène fréquemment à devoir se justifier. Les propos qui suivent révèlent également le flottement voire le cloisonnement pouvant exister, au sein même d'une fédération, entre les niveaux régional et national quant à la définition des missions d'un CTS. Si ce constat confirme que la gouvernance entre l'échelon central et les organisations territoriales d'une fédération demeure complexe à assurer (Bayle, 2010), il témoigne surtout du manque de dialogue existant parfois entre les diverses parties prenantes impliquées dans le suivi des CTS. Cette insuffisance, parce qu'elle rend difficile la fixation d'objectifs clairs et connus, est préjudiciable à la démarche d'appréciation du personnel (Trépo et al., 2002) :

"Pour cette année, j'ai été sollicitée au niveau national, j'ai finalement demandé à ce que ce volet national soit restreint parce que déjà ça faisait beaucoup de choses à faire et parce que moins je suis présente sur la région, moins mon président régional est conciliant... et donc du coup ça fait partie des choses auxquelles il faut veiller... » (un CTR).

S'agissant de leur mobilité interne, la plupart estiment là encore que leur changement de fonctions comme le maintien de leur placement auprès d'une fédération, dépendent plus des décisions du DTN et des dirigeants sportifs que de leur supérieur hiérarchique. En ce sens, la possibilité permise par les textes de mettre fin à leur mission avant le terme des quatre années, bien qu'il s'agisse d'agents titulaires de la fonction publique d'État, rend la gestion individuelle de leur carrière très chaotique voire exposée « au fait du prince » ou à l'arbitraire lorsque des indicateurs concrets tels que des contre-performances sportives obtenues par le groupe d'athlètes encadré par exemple, n'expliquent pas toujours le changement de fonctions.

Les CTS estiment ainsi avoir peu de prise sur ce processus de gestion individualisée de leur trajectoire professionnelle car il dépend non seulement de la division interne du travail établie par chaque DTN au sein d'une fédération mais aussi de l'appréciation que ce dernier a de leur travail. Évoquant l'opacité et l'absence de dialogue parfois quant aux décisions adoptées, les CTS peinent à expliquer et prévoir objectivement leur accession ou non à différents types de missions.

Partant de ce constat et exprimant par ailleurs clairement le principe d'une hiérarchisation des tâches qu'ils peuvent avoir à remplir, certains avouent subir ce qu'ils perçoivent comme étant des «inversions de carrière » lorsque de nouvelles affectations leur sont proposées. 
Les missions des CTS étant elles aussi marquées par des différences de prestige (Hughes, 1996), celles de niveau national, d'encadrement d'équipes seniors et d'athlètes masculins sont les plus recherchées ce qui met directement les CTS en concurrence les uns avec les autres pour les obtenir. À l'inverse, intervenir sur le seul échelon régional sur des fonctions de détection et d'encadrement d'équipes de jeunes ou de développement de la pratique peut être vécu comme une moindre reconnaissance de ses qualités :

« De 2000 à 2009, j'ai pu faire partie des équipes nationales et là dernièrement, j'ai été affectée en région... alors c'est vrai que moi je n'ai pas eu une ascension professionnelle classique parce que j'ai commencé entre guillemets par "le haut » et là je redescends en région. J'aimerais pouvoir retourner sur les missions nationales parce que c'est le truc quand même qui me dope le plus, parce qu'on a plus de moyens pour pouvoir fonctionner, je m'adresse à un public qui m'intéresse le plus, que je peux plus exploiter... mais ça, ça ne dépend pas de moi, ça dépend du futur DTN, donc, s'il ne veut pas de moi, il ne voudra pas de moi...» (une CTR).

De plus et au-delà du caractère moins prestigieux des missions régionales, n'exercer que celles-ci peut également être perçu comme rendant plus difficile toute perspective d'évolution de carrière et de mobilité interne choisie. C'est alors un sentiment de plafonnement subjectif de carrière dont il a été montré qu'il influe sur la motivation et le bien-être au travail, qui peut être ressenti par certains CTS, l'élément essentiel dans ce cas étant la perception qu'ont les travailleurs d'être restés trop longtemps sur leur poste, d'être peu motivés par les enjeux liés à leur fonction, de mériter une mobilité, etc. (Marc, 2008, p.102). Cet extrait d'entretien souligne aussi que la valorisation puis la reconnaissance de son travail auprès non pas du supérieur hiérarchique direct mais là encore, du DTN, peuvent être complexes à assurer et ce, en raison de deux principaux facteurs. La qualité et fréquence très variables des interactions que les CTS disent entretenir avec les DTN (et les dirigeants sportifs, $c f ., 2-2$ ) d'une part. Or, la nature des relations avec l'un des principaux appréciateurs du travail peut également conditionner les risques de démotivation (Garant et al., 2010). Ce sentiment, d'autre part, peut être renforcé par le fait que les DTN n'adoptent pas eux-mêmes des comportements identiques en matière de gestion des CTS placés auprès d'une fédération. Si certains enquêtés ont mentionné ne pas avoir été réunis par leur DTN depuis plus d'un an, dans d'autres fédérations, ces derniers pratiquent des entretiens avec les CTS afin de clarifier leurs missions au regard de la stratégie fédérale, de fixer et d'évaluer leurs objectifs et de déterminer leurs besoins, notamment, de formation.

Ainsi et face à cette hétérogénéité des démarches participatives des DTN et à ce qui est perçu comme une absence de transparence des décisions prises, des interviewés déplorent fortement l'absence de management des ressources humaines pour l'avancée de la carrière :

"Il y a un truc que je trouve très dur dans notre métier, c'est que personne ne vous interroge sur votre évolution de carrière... ce que je trouve très difficile et très dur, c'est que ce sont les événements qui font que vous n'évoluez pas ou oui... il y a un problème parce qu'en fin de compte, on s'aperçoit qu'on est livré à nous-mêmes... » (un CTR).

Les perspectives d'évolution statutaire existent toutefois depuis peu avec la création du corps de conseiller technique et pédagogique supérieur (CTPS) ${ }^{12}$, création dont le « caractère exceptionnel » a été souligné tant par le Ministre des Sports de l'époque (J.F. Lamour)

${ }^{12}$ Décret n²004-272 du 24 mars 2004 relatif au statut particulier des conseillers techniques et pédagogiques supérieurs. 
que par les syndicats «dans un contexte de réduction massive du nombre des corps de la fonction publique de l'État» (Ministère de la Jeunesse, des Sports et de la Vie Associative et CNOSF, 2006, p. 9). Les travaux menés lors des États Généraux du Sport en 2002 avaient souligné la nécessité d'accompagner la valorisation des compétences par une réforme de l'architecture des corps techniques et pédagogiques du Ministère des Sports. Deux arguments avaient alors milité en faveur de cette création : «la nécessité de prendre en compte l'excellence du travail effectué par une fraction des professeurs de sport, notamment à la tête des directions techniques des fédérations sportives, des équipes nationales ou encore des services déconcentrés et des établissements nationaux du MJSVA; l'intérêt de proposer de nouveaux types d'activités à des personnels techniques et pédagogiques ou encore à des étudiants afin de s'assurer du concours d'agents amenés à jouer un rôle technique de premier plan au sein du MJSVA et de les fidéliser » (MJSVA et CNOSF, 2006, p. 9).

Dix ans après la constitution de ce corps, les désillusions peuvent être grandes pour les CTS estimant qu'il n'existe que peu de transparence dans les modalités d'instruction des rapports des acquis de l'expérience professionnelle. Par ailleurs, le changement de corps, lorsqu'il est acquis, n'entraîne pas automatiquement une mobilité fonctionnelle qui continue souvent de dépendre des décisions des DTN.

Il apparaît enfin que les formes adoptées de suivi administratif des CTS varient, elles aussi, fortement d'un Directeur régional à un autre. Par ailleurs et au regard des éléments soulignant le rôle déterminant du DTN dans la définition des parcours professionnels, les initiatives prises par le Directeur régional pour assurer ce suivi sont perçues comme vides de sens :

"CTS, c'est un statut qui est très très bizarre, c'est-à-dire que l'on rend des trucs à la DRJSCS mais moi, quand j'étais affecté à la DR d'Ile-De-France je n'y mettais jamais les pieds et le Directeur n'avait aucune exigence administrative... peut-être une réunion, et encore, des nouveaux chaque année, je n'ai pas le souvenir qu'on avait des réunions... En Aquitaine le Directeur fait plus de suivi sur les CTS...» (un CTN).

Si les CTS le considèrent ainsi comme faiblement porteur d'enjeux, ils se voient désormais contraints de suivre le mouvement amorcé par les réformes de modernisation de l'État. L'autonomie professionnelle des CTS est actuellement menacée par l'avènement d'un «État évaluateur» (Paradeise, 2008) dont atteste la mise en place d'un outil de suivi de leur activité, le «CTS Web ». Destiné à ce qu'ils renseignent hebdomadairement le temps respectivement consacré aux quatre actions du Programme Sport, cet outil de gestion vise plus, pour l'administration centrale, à formaliser et à repérer les modes d'organisation du travail de ces agents qu'à normaliser leurs pratiques professionnelles.

Malgré le découplage régulier et constaté entre instruments et pratiques professionnelles (Dent et $a l ., 2004)$, il s'agit là d'une évolution que les CTS interprètent comme un mouvement de disciplinarisation. Traditionnellement attachés à leur autonomie professionnelle, certains, comme la principale organisation syndicale représentant leurs intérêts - le Syndicat National des Activités Physiques et Sportives (SNAPS) ${ }^{13}$-, se montrent très réservés voire réticents vis-à-vis de cette initiative. D'autres y voient l'opportunité de faire remonter auprès de l'administration centrale des informations sur l'exercice concret des missions :

${ }^{13}$ Le SNAPS considère que « l'outil n'est en l'état pas adapté à l'exercice de nos missions; sa conception relève d'un « idéal rationalisateur ». Or les spécificités techniques et culturelles de chaque fédération, sa taille et l'effectif des CTS rendent toute visée standardisante totalement illusoire », in SNAPS Infos, 96, octobre 2013, p. 19. 
«Cela a été mal accueilli je pense parce que c'est du flicage, c'est sûr... Après, on en a discuté, on s'est dit : " bon, il doit y avoir quand même des avantages à cet outil ». Et au final, on y voit un intérêt. Vu que c'est un outil qui va permettre de faire des croisements, je suppose, au niveau du Ministère, notamment avec les lettres de mission, on s'est dit que finalement, 1607 heures pour nous, qui est le quota annuel, on va prouver que c'est très très vague. Nous, on fonctionne à la mission, on ne regarde pas le temps qu'on passe à une mission. On est susceptible de partir pendant une semaine en compétition, même deux semaines de suite, donc, qu'est-ce qui se passe au niveau de la récup? Donc, on met tout cela sur l'agenda qu'on remplit et puis ça peut faire évoluer peut-être les esprits des gens qui vont regarder, qui sont peut-être un petit peu moins sur le terrain avec nous et qui, peut-être, ne se doutent pas de la charge de travail qu'on peut avoir à un certain moment. » (un CTN).

S'il n'est pas encore possible d'apprécier objectivement tous les effets concrets de cet outil dans la mesure où il n'est opérationnel que depuis septembre 2012, les modes de régulation du travail des CTS sont ainsi et eux aussi, en train de migrer d'une forme « professionnelle » à une forme « bureaucratique » au sens anglo-saxon (Abbott, 1988).

L'ensemble de ces éléments rend notamment complexe la procédure de leur évaluation aussi bien individuelle que collective.

\subsection{Des parcours professionnels entachés par des conflits d'intérêts}

Outre le fait que les DTN apparaissent comme les arbitres essentiels des parcours des CTS, leur situation professionnelle est également dépendante des configurations fédérales et des stratégies des dirigeants sportifs. Plusieurs points en attestent.

Le métier de CTS, qualifié de «métier de passion et de conflits» avec les dirigeants fédéraux qui déploient fréquemment des velléités de contrôle de l'activité de ces agents, les place dans des situations professionnelles pouvant devenir complexes voire douloureuses. Les CTS rencontrés déplorent ces tentatives d'instauration de rapports « serviciels » dont ils sont l'objet et qui nuisent à l'ambiance au travail, dimension importante là encore du maintien de la motivation et de l'efficacité des professionnels (Giauque et $a l ., 2008$ ) Dans ce cas, s'ils cherchent systématiquement à contrôler la définition de leur activité - la revendication de leur autonomie d'action étant ici aussi placée au cœur du débat (Paradeise, 2008) ${ }^{14}$-, ils avouent toutefois qu'ils ne disposent pas toujours des ressources pour s'extraire de ces stratégies de domination. Alors que les représentants des organisations syndicales admettent que l'administration centrale peine à gérer les situations de conflits que cela engendre avec les dirigeants sportifs, ils indiquent par ailleurs qu'elles se soldent par une réaffectation de missions le plus souvent subie par les CTS :

"Quand un président de fédération ne veut plus d'un cadre, entraîneur national ou autre, l'État s'évertue à lui trouver, comme il est fonctionnaire, des points de chute ici ou là; ce qui est de plus en plus compliqué parce que le ministère n'a pas un énorme éventail de possibilités de niches pour positionner les uns et les autres »(un représentant syndical).

"On se retrouve dans un statut bizarre où on a réellement l'impression que la seule à qui on va rendre des comptes c'est la fédération... c'est un peu vrai aussi parce que

${ }^{14}$ La défense de cette autonomie professionnelle constitue une revendication permanente pour les représentants syndicaux. Lire par exemple Lernould C., " L'autonomie ou la mort... », SNAPS Infos, 94, p. 13-14. 
les seuls qui à un moment donné peuvent dire "Non on n'a plus trop besoin de lui » ou qui nous encouragent à partir pour des raisons $X$ ou $Y$ c'est quand même eux à la fédération, c'est eux qui au quotidien contrôlent notre travail... donc la DR...elle est loin, elle met une note administrative (sourire) mais des fois je me demande comment, comment le DR il peut savoir exactement ce que je fais » (un CTN).

Les CTS, en étant des marginaux sécants au croisement de plusieurs organisations (la DRJSCS, le comité régional et la fédération sportive), sont ainsi affranchis de liens exclusifs avec le Directeur régional. Si cette situation leur confère une liberté certaine, elle consacre, on l'a vu, l'influence du DTN mais aussi celle des dirigeants sportifs. Il est alors difficile de concevoir des parcours de carrière coordonnés mais l'extrait d'entretien qui précède montre aussi que cette situation influe également sur l'évaluation individuelle de leur action.

Dans ce marché du travail très particulier effectivement où les règles de fonctionnement sont avant tout régies par des liens d'interconnaissance et peu par des critères tels que le diplôme, la formation ou l'ancienneté, les obligations qui incombent désormais aux administrations de mettre progressivement fin à la notation au profit d'entretiens professionnels destinés à mieux apprécier le mérite des agents, ne sont guère porteuses d'espoirs pour les CTS. Elles sont par ailleurs fortement contestées par les organisations syndicales. Les motifs évoqués pour refuser ces avancées de carrière à partir de l'évaluation du mérite concernent les indicateurs d'appréciation de ce dernier. Pour les CTS et les représentants syndicaux, leur définition même consacrerait déjà une forme d'arbitraire :

"Comment comparer un CTS médaillé olympique dans une fédération qui a décidé de faire une politique de haut niveau en y mettant les moyens très tôt avec un CTS qui lui, rentre dans une fédération où il y a tout à faire et dont les résultats n'arriveront peut-être que dans 10 ou 15 ans? Comment vous les notez? Aujourd'hui, la notation des cadres techniques par le mérite, c'est une utopie » (un CTN).

Cet extrait d'entretien renvoie également au fait que la démarche d'appréciation du travail des CTS devrait être située et prendre en compte les différences de contextes et le fait que ces agents occupent des postes distincts, dimensions considérées comme plus déterminantes que les outils de gestion des ressources humaines eux-mêmes (Garant et al., 2010). Or, les modalités actuelles de notation des CTS adoptées par les Directeurs régionaux procèdent plus du modèle d'évaluation impersonnelle qui homogénéise la reconnaissance des apports individuels en tenant compte de standards tels que l'âge et l'ancienneté (Baudry et Dubrion, 2005), démarche qui obère ainsi les contributions individuelles et le contexte dans lequel ces dernières se déploient. Si les CTS considèrent que ce mode de notation ne constitue pas un outil fiable pour bien apprécier leur travail, ils ne le récusent pas pour autant. Au regard de la manière connue de tous dont il est pratiqué, il est perçu, au contraire des décisions prises par les DTN et les dirigeants sportifs qu'ils estiment entachées d'arbitraire et de biais de jugement, comme garantissant une certaine « égalité » de traitement.

D'autres arguments sont également évoqués pour décrédibiliser ce processus d'évaluation, non plus individuelle mais collective, de la performance induite par la LOLF. Alors que le travail demandé à l'ensemble des services de l'État dans cette loi consiste à définir des objectifs et indicateurs de résultat, les CTS et les représentants syndicaux indiquent, dans ce secteur d'action publique comme dans d'autres en France (Winiki, 2006), que la performance attendue ne dépend pas uniquement de l'action du Ministère des Sports mais implique d'autres administrations publiques, opérateurs ou acteurs divers dont les instances fédérales : 
« La LOLF nous convient parfaitement à condition d'y intégrer le fait que les indicateurs sont des indicateurs soumis aux aléas de partenaires non administratifs et non contrôlables. Nous ne sommes pas contre, à condition qu'il soit clairement expliqué dans la LOLF que les indicateurs ne peuvent pas être pris au pied de la lettre puisqu'ils dépendent de la politique et du fonctionnement d'opérateurs sur lesquels l'État n'a aucun moyen » (un représentant syndical).

Les interactions permanentes avec les dirigeants sportifs ont d'autres conséquences sinon contradictoires du moins ambivalentes sur la gestion des parcours professionnels des CTS. À ce titre, si leur position institutionnelle les expose aux velléités de domination des élus fédéraux, elle leur offre également la possibilité d'en retirer des avantages, non prévus statutairement, en termes de rémunération. Certaines fédérations en effet accordent une prime aux divers CTS dont le montant n'est, par ailleurs, parfois pas volontairement communiqué au Ministère des Sports (Cour des Comptes, 2013).

Ceci accentue non seulement la complexité du partenariat entre celles-ci et l'État mais renforce aussi l'ambiguïté précédemment évoquée de la gestion des carrières des CTS. Les stratégies de ces organisations multiplient, en effet, les possibilités de différenciation de leurs trajectoires professionnelles en fonction, plus particulièrement, de la structure économique de la discipline sportive en question. Cela suscite immanquablement des interrogations de la part de la Cour des Comptes contestant la régularité juridique de ces « compléments indemnitaires » $(2009 ; 2013)$, mais aussi des amertumes et des jalousies dans la profession au regard des fortes inégalités de traitement salarial entre agents du même corps. Cette différenciation des trajectoires des CTS conduit à une segmentation de leur espace professionnel. À la distinction des missions propre aux CTS, se superpose désormais la frontière entre ceux pouvant uniquement compter sur leur traitement d'agent public et les autres (Honta et Julhe, 2013).

\subsection{Des situations professionnelles exposées aux ambiguiités du partenariat entre l'État et le mouvement sportif}

Les résultats présentés ici montrent que les préceptes managériaux contenus dans les textes ne sont pas ceux qui guident les pratiques concrètes de gestion de la carrière des CTS. La position que le Ministère des Sports souhaite conserver dans le secteur sportif et les principes structurant ses rapports avec les fédérations sportives interfèrent avec les axes de réformes adoptées et constituent la principale explication à ces décalages.

Le fait que les CTS n'exercent pas leur activité au sein des services de l'État mais, le plus souvent, des structures d'encadrement des athlètes, des comités sportifs régionaux ou des fédérations, expose leur supérieur hiérarchique qu'est le Directeur régional à procéder à un « management lointain » (Pochard, 2008) de ces personnels. Cela interroge les possibilités effectives non seulement du suivi de ces agents mais aussi de leur évaluation. Alors que l'efficacité du dispositif d'individualisation des carrières doit reposer sur un avis averti du chef de service, l'incertitude informationnelle dans laquelle ce dernier se trouve fait qu'il se contente souvent, lors de la notation des CTS, de quelques formules convenues qui le protègent, dans le même temps, des recours aux organisations syndicales. Il accorde ainsi, le plus souvent, la même notation à tous en fonction des critères de la grille pour ne faire aucune discrimination de plus au regard de l'hétérogénéité des contextes d'exercice des 
missions et des pratiques de management adoptées par les divers DTN. Il est intéressant de préciser à ce titre que la note de service relative à l'évaluation des professeurs de sport alerte les chefs de service sur le fait que «la rubrique «fonctions et domaines d'intervention » de l'agent au cours de l'année doit être remplie avec précision et en relation avec les missions statutaires du corps concerné; trop souvent cette rubrique n'est pas remplie ou est imprécise $\gg^{15}$.

Le Directeur régional, pour organiser cette gestion, doit en effet passer par des intermédiaires que sont le DTN et les dirigeants sportifs qui disposent, en la matière, d'une véritable influence. Une telle situation obère clairement les possibilités pour le Ministère des Sports d'apprécier objectivement ce que font ces agents et dans quelle mesure ils participent à la performance désormais attendue de la politique sportive nationale. Ainsi, la compréhension des ressorts de l'évaluation des compétences, de la carrière, du potentiel des CTS demeure encore et ici aussi une énigme (Chanut et Rojot, 2011), dans la mesure où le mode de gestion de leur parcours professionnel juxtapose, mais n'articule pas, une idéologie du secteur public traditionnellement égalitaire et rétive à toute forme de différenciation que traduit leur mode de notation par le Directeur régional et une logique contractuelle qui incite à rendre des comptes au DTN et aux dirigeants fédéraux mais à les rendre sans que les règles du contrat soient toujours lisibles et transparentes.

Si l'outil que constitue le «CTS Web» permettra très probablement de mieux cerner les pratiques professionnelles des CTS à l'avenir et leur distribution au sein des quatre actions du Programme Sport, restent que les indicateurs de résultats actuellement retenus pour apprécier la performance de cette administration et de ses agents posent question.

D'une part parce que les conditions de travail des CTS au sein des diverses fédérations dépendent aussi des ressources de celles-ci. Outre les aides de l'État qu'elles reçoivent, plusieurs d'entre elles sont parvenues ces trente dernières années à diversifier leurs recettes grâce notamment à des partenariats avec des entreprises. Ces capacités financières leur permettent de recruter leurs propres salariés sous contrat de droit privé qui peuvent constituer de précieux collaborateurs pour les CTS dans le processus aussi bien de fabrication de l'élite sportive que du développement de la discipline. D'autre part car de nombreux autres acteurs, dont la définition de leurs compétences respectives fait d'ailleurs défaut, participent à l'organisation du sport et à l'atteinte donc des indicateurs de performance. Qu'ils s'agissent des entreprises, des clubs, des fédérations, des collectivités territoriales ou d'autres Ministères, leurs formes de mobilisation sont désormais plurielles et contribuent indéniablement tant à la promotion du sport de haut niveau que du sport pour le plus grand nombre. Dans ce contexte, il demeure aujourd'hui extrêmement difficile pour le Ministère des Sports de connaître précisément à quelle hauteur il contribue aux résultats des diverses actions de son Programme Sport.

Ces constats accentuent sans doute le déficit de légitimité dans lequel se trouve cette administration en France. Alors que l'effort de nécessaire maîtrise des finances publiques engagé par l'État contraint le Ministère à la réduction des crédits du Programme Sport, le président du Comité National Olympique et Sportif Français (CNOSF) et certains présidents de fédérations sportives revendiquent aujourd'hui un autre modèle de gouvernance du sport en général, du sport de haut niveau en particulier (CNOSF, 2014). Leurs attentes

\footnotetext{
${ }^{15}$ Note de service NDRH/DRH1C/2013/278 du 10 juillet 2013, op.cit., p. 3.
} 
respectives ne se recoupent toutefois qu'en partie. Alors que le premier souhaite aujourd'hui que l'État lui transfère le pilotage de la politique publique du sport de haut niveau - proposition à laquelle s'oppose le principal syndicat des $\mathrm{CTS}^{16}$ - les seconds attendent plutôt du Ministère des Sports qu'il leur permette de gérer directement les carrières des CTS (Cour des Comptes, 2009).

Si ces revendications sont récurrentes en France, régulièrement relayées par la presse nationale par ailleurs, le Ministère des Sports refuse, pour l'heure, d'y répondre favorablement. Il profite, à ce titre, de la très forte hétérogénéité aussi bien des ressources que du processus de professionnalisation des diverses fédérations sportives. Celle-ci explique que celles qui peinent à diversifier leurs recettes d'activité restent encore sous dépendance étatique. Cette diversité des configurations fédérales empêche ainsi et plus généralement ces acteurs sportifs d'avancer unis dans leurs revendications voire contestations à l'égard de l'État. Dans ce contexte, la ressource que constituent les CTS permet incontestablement au Ministère des Sports de «peser » encore dans le pilotage de cette politique et dans ses rapports au mouvement sportif.

\section{Conclusion}

Le discours des CTS conforte le constat réalisé par plusieurs travaux (Chanut, 2008; Cousin, 2008), ayant montré, à partir d'enquêtes portant à la fois sur les secteurs public et privé, l'insatisfaction des cadres concernant la gestion de leur carrière plus particulièrement ici car ces professionnels n'ont finalement que peu de retours sur ce qu'ils réalisent, principe pourtant essentiel de valorisation et de reconnaissance du travail. Alors que la qualité de l'échange entre appréciateurs et appréciés doit permettre de renforcer la confiance, la motivation individuelle et de fonder un « contrat mutuel » entre les parties (Dumond, 2006 ; Giauque et al., 2008; Palpacuer et al., 2010), ces éléments, dans le cas des CTS, sont encore très dépendants de la nature et de la fréquence des relations qu'ils entretiennent avec les DTN et les dirigeants sportifs.

Aussi, la gestion de leur parcours professionnel dépend plus aujourd'hui d'une logique fédérale et associative - déterminant le contexte mais aussi l'ambiance au travail - que d'une logique managériale telle que les textes la préconisent. La position administrative des CTS les expose en effet à la porosité des frontières entre deux « cultures » - celle de la fonction publique et celle du mouvement sportif - et finalement entre deux temporalités. Le premier de ces contextes, qui encadre juridiquement leur emploi, propose avant tout un raisonnement sur le temps long relatif à la carrière prise dans son ensemble; tandis que le second, qui définit le cadre d'exercice de leur travail, est davantage structuré autour de la saison ou de l'olympiade sportive et des exigences, le plus souvent à court terme, de performances sportives ou d'augmentation des effectifs de licenciés dans les clubs. Dans ce cas et lorsque les résultats ne sont pas considérés à la hauteur, notamment par les dirigeants sportifs, le biais de la recherche du coupable présent dans le monde de l'entreprise (Trépo et al., 2002), se retrouve également ici et conduit à rendre parfois imprévisibles les

\footnotetext{
${ }^{16}$ La Lettre de I'Officiel juridique du sport (2013), Jean-Paul Krumbholz : « Le sport haut-niveau doit rester un domaine partagé entre le ministère des Sports, les fédérations délégataires et le CNOSF », 74, juin.
} 
changements survenant au cours de leur carrière. Si les CTS s'en trouvent désabusés et démotivés, cette forme d'imprévisibilité, toutefois, apparaît contrebalancée par un faible degré d'irréversibilité dans le sens où de nouvelles opportunités de mission peuvent s'offrir à eux en raison de la pluralité des parties en présence et des changements, fréquents dans certaines fédérations, de dirigeants sportifs et de DTN, ces derniers étant eux-mêmes exposés à « l'action corrosive de l'acide chlorhydrique du monde associatif » (Ettore, 2012, p. 47).

Plus généralement, si ces éléments expliquent que les évolutions relatives à la gestion des ressources humaines prévues dans le cadre des réformes de modernisation de l'État n'ont produit que des effets limités, la situation professionnelle des CTS pourrait connaître des évolutions dans un proche avenir. La Cour des Comptes (2013) a rendu public un rapport invitant le Ministère des Sports à rechercher une meilleure efficience dans les moyens qu'il consacre à sa politique sportive et à revoir les principes de gestion des CTS. Les modalités du suivi et du contrôle de leur activité, les critères historiquement adoptés de leur placement auprès du mouvement sportif, les compléments de rémunération versés par les fédérations et l'ambiguïté de leur position administrative sont des points, de nouveau, vivement critiqués (Lavaure et Watrin, 2011). Alors que le gouvernement actuel lance une nouvelle réforme de l'État comportant là encore un volet sur la fonction publique - la « Modernisation de l'action publique » (Pêcheur, 2013) - la nouvelle ministre des Sports, Najat Vallaud-Belkacem a annoncé « un nouveau départ pour le sport français » dans un climat de concertation traditionnellement ambivalent et désormais tendu avec le mouvement sportif ${ }^{17}$. Dans ce contexte de réforme et d'incertitude que certains chercheurs estiment propice pour engager simultanément une démarche prospective des dispositifs de management des ressources humaines afin de recréer du sens au sein d'une administration (Giauque et Caron, 2004 ; Chanut et Rojot, 2011), le SNAPS défend plutôt le maintien d'un statu quo concernant la gestion des carrières des CTS, la position du Ministère restant, quant à elle, encore méconnue.

\footnotetext{
${ }^{17}$ Allocution réalisée le jeudi 17 avril 2014 devant le conseil d'administration du CNOSF.
} 


\section{Bibliographie}

ABBOTT A., (1988). The System of Professions. An Essay on the Division of Expert Labor. The University of Chicago Press, Chicago.

AUCOIN P., (1990). Administrative Reform in Public Management: Paradigms, Principles, Paradoxes and Pendulums. Governance 3, 115-137.

BAUDRY B., DUBRION B. (2005), Quels modèles d'évaluation du travail ? Travail et Emploi, 104, 7-18.

BAYLE E. (2010), La gouvernance des fédérations d'associations chargées d'une mission de service public. Le cas des fédérations sportives françaises. Politiques et Management Public, 27(1), 121-142.

BERTÉŻ̀NE S. et DUBRION B., (2013). « Moderniser » les pratiques d'évaluation du travail dans la fonction publique : Analyse exploratoire du cas d'un hôpital public. Formation emploi 121, 83-105.

BEZES P., (2008). Le tournant néomanagérial de l'administration française In O. BORRAZ et V. GUIRAUDON (dir.), Politiques publiques, Presses de Sciences Po, Paris, 215-254.

BOURGON J., (2009). Programme Review: The Governement of Canada's Experience Eliminating the Deficit, 1994-99: a Canadian Case Study. Institute for Governement, London.

CHANUT V., (2008). Manager versus expert? Ou les avatars de la gestion personnalisée des cadres dans une grande administration technique. Revue française d'administration publique 128, 703-716.

CHANUT V. et ROJOT J., (2011). Réinventer l'évaluation. Les nouvelles règles du jeu RH dans une administration publique. Management \& Avenir 49, 195-222.

CHEMLA-LAFAY A., (2008). Professionnaliser la GRH dans la fonction publique de l'État en France, une incantation sans effets réels. Politiques et Management Public 26 (2), 111-135.

CHEVALLIER J., (2010). Révision générale des politiques publiques et gestion des ressources humaines. Revue Française d'Administration Publique 136, 907-918.

COMITÉ NATIONAL OLYMPIQUE ET SPORTIF FRANÇAIS (CNOSF), (2014). Projet du CNOSF pour le sport français. Une ambition : "Passer d'une nation de sportifs à une nation sportive ». Une clé : "Rénover le modèle sportif français, 9 janvier 2014 (www.franceolympique.com).

COUR DES COMPTES, (2009). L'État et les fédérations sportives face aux mutations du sport. Rapport d'activité.

COUR DES COMPTES, (2013). Sport pour tous et sport de haut niveau : pour une réorientation de l'action de l'État. Rapport thématique.

COUSIN O., (2008). Les cadres à l'épreuve du travail. PUR, Rennes.

DENT M., O’NEILL M. and BAGLEY C., (1999). Professions: New Public Management and the European Welfare State. University Press, Staffordshire.

DREYFUS F., (2006). Servir l'État, un idéal encore moderne? Pouvoirs 117, 5-15.

DUMOND J.P., (2006). L'appréciation des personnels. Évaluer n'est pas expliciter. Revue française de gestion 162, 21-34.

ETTORE G., (2012). Rapport d'information sur la gouvernance des fédérations sportives. Assemblée Nationale, Paris.

FERLIE E., PETTIGREW A., ASHBURNER L. and FITZGERALD L., (1996). The New Public Management in Action. Oxford University Press, Oxford.

GARANT M., DELOBBE N., DUJARDIN C. et OIRY E., (2010). L'évaluation du personnel : la Gestion des Ressources Humaines peut-elle contribuer au développement professionnel? In L. PAQUAY et al., L'évaluation, levier du développement professionnel? De Boeck, Bruxelles, 67-78.

GIAUQUE D. et CARON J.D., (2004). Stratégies des réformes administratives et impacts sur la gestion des ressources humaines. Une comparaison Suisse-Canada. Politiques et Management Public 22 (3), 63-80.

GIAUQUE D. et al., (2008). Les leviers de la performance individuelle et collective dans les organisations publiques Suisses : l'importance d'un pilotage participatif. Revue française d'administration publique 128, 785-798. 
GROSSMAN E., (2007). Les groupes d'intérêt en France In P. PERRINEAU et L. ROUBAN (dir.), La politique en France et en Europe, Presses de Sciences Po, Paris, 155-184.

HONTA M., (2009). Révision générale des politiques publiques et réforme du Ministère de la Santé et des Sports : la justification par la performance. Revue Juridique et Économique du Sport 91, 7-25.

HONTA M. et JULHE S., (2013). Les professions du secteur public saisies par la privatisation. Le cas des Conseillers Techniques Sportifs. Gouvernement et action publique 1, 63-87.

HOOD C. and LODGE M., (2004). Competency, Bureaucracy and Public Management Reform: a Comparative Analysis. Governance 17 (3), 313-333.

HORTON S., HONDEGHEM A. and FARNHAM D., (2002). Competency Management in the Public Sector: European Variations on a Theme. IOS, Amsterdam.

HOULIHAN B. and GREEN M., (2009). Modernization and Sport: the reform of sport England and UK Sport. Public Administration 87 (3), 678-698.

HUGUES E. C., (1996). Le regard sociologique. Essais choisis. EHESS, Paris.

JEANNOT G., (2005). Gérer les carrières des cadres de la fonction publique? Revue Française d'Administration Publique 116, 553-560.

JOBERT B. et MULLER P., (1987). L'État en action : politiques publiques et corporatismes. PUF, Paris.

KEELER J., (1987). The Politics of Neo-Corporatism in France: Farmers, the State and Agricultural PolicyMaking in the Fifth Republic. Oxford University Press, Oxford.

LAPIN J., (2009). Performance et fonction publique de l'État: les récentes réformes. Revue Française d'Administration Publique 131, 601-614.

LAPSLEY I. et MIDWINTER A., (2010). Moderniser l'État : les aspects financiers des programme reviews au Royaume-Uni. Revue Française d'Administration Publique 136, 821-835.

LAVAURE P. et WATRIN D., (2011). Rapport relatif aux missions des cadres techniques sportifs placés auprès des fédérations sportives. Inspection générale de la Jeunesse et des Sports.

LEMOYNE DE FORGES J-M., (2009). Quelle influence communautaire sur l'avenir du modèle français de fonction publique? Revue Française d'Administration Publique 132, 701-710.

MARC D., (2008). Le plafonnement des carrières : le cas des techniciens de l'environnement, entre contrainte réglementaire et manque de reconnaissance. Politiques et Management Public 26 (2), 99-110.

MASSEY A., (2010). Les programme reviews au Royaume-Uni et la question de la mesure de la performance. Revue Française d'Administration Publique 136, 837-855.

MÉDA D., (2010) Comment mesurer la valeur accordée au travail? Sociologie 1, 121-140.

MINISTÈRE DE LA JEUNESSE, DES SPORTS ET DE LA VIE ASSOCIATIVE ET COMITÉ NATIONAL OLYMPIQUE ET SPORT FRANÇAIS, (2006). 2002-2006. La mise en œuvre des mesures pour le développement du sport à l'issue des États Généraux du Sport.

OCTOBRE S., (1999). Profession, segments professionnels et identité. L'évolution des conservateurs de musées. Revue française de sociologie 4 (2), 357-383.

PALPACUER F., VERCHER C., TESSIER N. et BOURDON N., (2010). Sous l'épée de Damoclès : l'évaluation des cadres, entre performance individuelle et esprit d'équipe. Management \& Avenir 35, 15-32.

PARADEISE C., (2008). Autonomie et régulation : retour sur deux notions clés In T. LE BIANIC et A. VION (dir.), Action publique et légitimités professionnelles, LGDJ, Paris, 289-296.

POCHARD M., (2003). Perspectives pour la fonction publique, Conseil d'État - Rapport public. La Documentation française, Paris.

POCHARD M., (2008). Nouvelles perspectives pour la fonction publique. Politiques et Management Public 26 (2), 3-13.

POllitT C. and BOUCKAERT G., (2004). Public Management Reform. A Comparative Analysis $2^{\text {nd }} e d$. Oxford University Press, Oxford.

PÊCHEUR B., (2013). Rapport à Monsieur le Premier ministre sur la fonction publique, 29 octobre. 
SILICANI J-L., (2008). Livre blanc sur l'avenir de la fonction publique, Rapport au Ministère du budget, des comptes publics et de la fonction publique. La Documentation française, Paris.

TALBOT C., (2003). La réforme de la gestion publique et ses paradoxes : l'expérience britannique. Revue Française d'Administration Publique 105-106, 11-24.

TREPO G., ESTELLAT N. et OIRY E., (2002). L'appréciation du personnel, mirage ou oasis? Éditions d'Organisation, Paris.

VALLEMONT S., (1999). La gestion des ressources humaines dans l'administration. Rapport pour le Ministre de la fonction publique, de la réforme de l'État et de la décentralisation. La Documentation française, Paris.

WASS D., (1983). The Public Service in Modern Administration. Public Administration 61 (1), 7-20.

WINIKI P., (2006). Loi organique relative à la loi de finances (LOLF), gestion des ressources humaines et nouvelles compétences managériales. Revue Française d'Administration Publique 117, 117-130. 